\title{
Feasibility of deep-inspiration breath-hold PET/CT with short-time acquisition: detectability for pulmonary lesions compared with respiratory-gated PET/CT
}

\author{
Shozo Yamashita $\cdot$ Kunihiko Yokoyama • \\ Masahisa Onoguchi · Haruki Yamamoto • \\ Shigeaki Hiko $\cdot$ Akihiro Horita $\cdot$ Kenichi Nakajima
}

Received: 8 April 2013/Accepted: 18 September 2013/Published online: 23 October 2013

(C) The Author(s) 2013. This article is published with open access at Springerlink.com

\begin{abstract}
Objectives Deep-inspiration breath-hold (DIBH) PET/CT with short-time acquisition and respiratory-gated (RG) $\mathrm{PET} / \mathrm{CT}$ are performed for pulmonary lesions to reduce the respiratory motion artifacts, and to obtain more accurate standardized uptake value (SUV). DIBH PET/CT demonstrates significant advantages in terms of rapid examination, good quality of $\mathrm{CT}$ images and low radiation exposure. On the other hand, the image quality of DIBH PET is generally inferior to that of RG PET because of short-time acquisition resulting in poor signal-to-noise ratio. In this study, RG PET has been regarded as a gold standard, and its detectability between DIBH and RG PET studies was compared using each of the most optimal reconstruction parameters.

Methods In the phantom study, the most optimal reconstruction parameters for DIBH and RG PET were
\end{abstract}

S. Yamashita $\cdot$ H. Yamamoto $\cdot$ S. Hiko $\cdot$ A. Horita

Division of Radiology, Public Central Hospital of Matto

Ishikawa, 3-8 Kuramitsu, Hakusan, Ishikawa 924-8588, Japan

e-mail: y.shozo57@gmail.com

S. Yamashita $\cdot$ M. Onoguchi $(\bowtie)$

Department of Health Sciences, Graduate School of Medical

Sciences, Kanazawa University, 5-11-80 Kodatsuno, Kanazawa,

Ishikawa 920-0942, Japan

e-mail: onoguchi@staff.kanazawa-u.ac.jp

K. Yokoyama

PET Imaging Center, Public Central Hospital of Matto Ishikawa,

3-8 Kuramitsu, Hakusan, Ishikawa 924-8588, Japan

e-mail: yokoyama@mattohp.com

K. Nakajima

Department of Nuclear Medicine, Kanazawa University

Hospital, 13-1 Takara-machi, Kanazawa,

Ishikawa 920-8641, Japan determined. In the clinical study, 19 cases were examined using each of the most optimal reconstruction parameters. Results In the phantom study, the most optimal reconstruction parameters for DIBH and RG PET were different. Reconstruction parameters of DIBH PET could be obtained by reducing the number of subsets for those of RG PET in the state of fixing the number of iterations. In the clinical study, high correlation in the maximum SUV was observed between DIBH and RG PET studies. The clinical result was consistent with that of the phantom study surrounded by air since most of the lesions were located in the low pulmonary radioactivity.

Conclusion DIBH PET/CT may be the most practical method which can be the first choice to reduce respiratory motion artifacts if the detectability of DIBH PET is equivalent with that of RG PET. Although DIBH PET may have limitations in suboptimal signal-to-noise ratio, most of the lesions surrounded by low background radioactivity could provide nearly equivalent image quality between DIBH and RG PET studies when each of the most optimal reconstruction parameters was used.

Keywords Deep-inspiration breath-hold (DIBH) PET/CT · Respiratory-gated (RG) PET/CT ·

Pulmonary lesion $\cdot$ Reconstruction parameters

\section{Introduction}

Positron emission tomography/computed tomography (PET/CT) with ${ }^{18}$ F-fluorodeoxyglucose $\left({ }^{18} \mathrm{~F}-\mathrm{FDG}\right)$ can visualize human glycometabolism, and is widely used for the diagnosis of lesions and staging of diseases [1-3]. PET/ CT can provide more accurate anatomical locations than dedicated PET system. It is, moreover, advantageous for 
shortening an examination time over PET, and attenuation is accurately corrected using $\mu$-map calculated by Hounsfield units from CT images [4]. Recently, PET/CT is also used for radiotherapy and to assess the effectiveness of therapy [5-8]. However, misregistration between PET and CT images may occur because PET and CT data are acquired sequentially, and lesions detected by PET are not consistent with those detected by CT if the lesion is moved by the body motion, respiration and peristalsis. The motions result in unclear images and inaccurate standardized uptake value (SUV).

Deep-inspiration breath-hold (DIBH) PET/CT with short-time acquisition and respiratory-gated (RG) PET/CT are performed to reduce the respiratory motion artifacts, and to obtain more accurate SUV [9-17].

The signal-to-noise ratio of RG PET is better because of the longer time of acquisition than that with DIBH PET. The RG PET/CT can also be used for patients who cannot maintain breath holding for a long duration. However, device preparation and acquisition time are somewhat cumbersome and take a longer time, which may cause a burden to the patients or delay in study schedule. RG CT has major drawbacks in terms of high radiation exposure because of repeated cine mode scan and poor image quality due to the body motion of free breathing and low tube current time product.

While the image quality of DIBH PET is generally inferior to that of RG PET due to short-time acquisition, DIBH PET/CT has significant advantages in terms of its rapid examination, better $\mathrm{CT}$ image quality and low radiation exposure.

In our study, RG PET has been regarded as a gold standard, and its detectability between DIBH and RG PET was compared using each of the most optimal reconstruction parameters demonstrated in the phantom study. To the best of our knowledge, this is the first report on lesions providing equivalent image quality between DIBH and RG PET. In addition, no study to date has been conducted to determine the reconstruction parameters of DIBH PET on the basis of the optimal reconstruction parameters of RG PET.

\section{Materials and methods}

Phantom study

The National Electrical Manufacturers Association (NEMA) 2001 International Electrotechnical Commission (IEC) phantom (Data Spectrum Corp., Hillsborough, NC) was used for this study. This phantom consisted of a torso cavity, a removable lung insert, and 6 spheres. The inner diameters of these spheres were 10,13,17, 22, 28 and $37 \mathrm{~mm}$. They were filled with ${ }^{18} \mathrm{~F}$-FDG solutions of the same radioactivity concentration $(10.6 \mathrm{kBq} / \mathrm{mL})$, and the background $(\mathrm{BG})$ was set

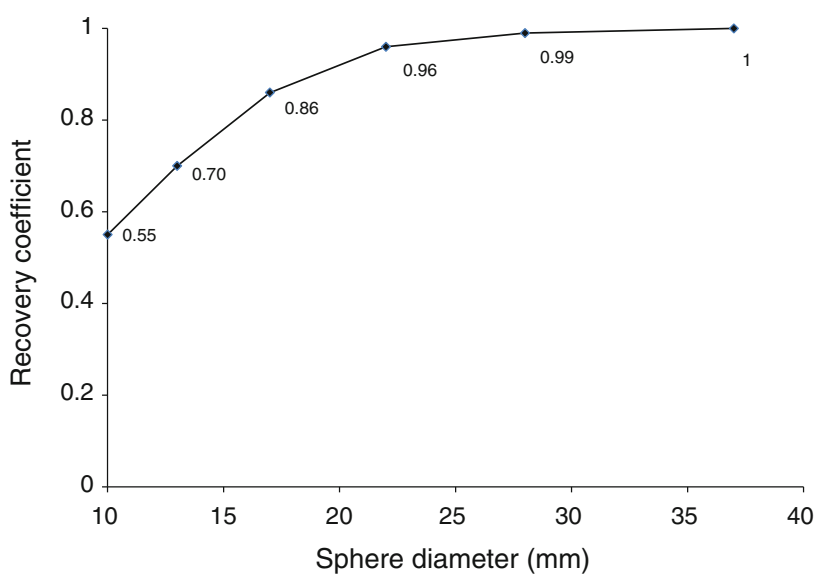

Fig. 1 The recovery coefficient (RC) of the PET/CT system used in this study. The RCs were determined referring to the Japanese Guideline for Oncology PET/CT. The phantom image was reconstructed by the optimal reconstruction parameters for RG PET

to $2.65,1.33 \mathrm{kBq} / \mathrm{mL}$ and none (air). They were scanned using a list-mode dynamic acquisition method. The most optimal reconstruction parameters for RG PET were determined referring to the Japanese Guideline for Oncology of FDG-PET/CT [18], and the phantom filled with $2.65 \mathrm{kBq} / \mathrm{mL}$ in the BG was used. Other phantoms were used to determine the optimal reconstruction parameters for DIBH PET. At our institution, ${ }^{18} \mathrm{~F}-\mathrm{FDG}$ is injected with radioactivity of $4.4 \mathrm{kBq} /$ $\mathrm{g}$, and RG and DIBH PET are performed at about $150 \mathrm{~min}$ after injection (physical decay to $39 \%$ ). If the percentage of injected radioactivity excreted to the bladder is $23 \%$ [19], and the percentage of the adipose tissue is $27 \%$ of the total body volume, the radioactivity of the mediastinum at $150 \mathrm{~min}$ after injection is estimated to be $1.81 \mathrm{kBq} / \mathrm{mL}(4.4 \mathrm{kBq} /$ $\mathrm{g} \times 1 \mathrm{~g} / \mathrm{mL} \times 0.39 \times 0.77 / 0.73=1.81)$, which is equivalent to $1.05 \mathrm{SUV}(0.77 / 0.73=1.05)$. Then, $1.33 \mathrm{kBq} / \mathrm{mL}$ is equivalent to $0.77 \mathrm{SUV}$. In addition, $10.6 \mathrm{kBq} / \mathrm{mL}$ is equivalent to $6.16 \mathrm{SUV}$, and the SUV of the $10-\mathrm{mm}$ sphere is equivalent to 3.39 SUV because the recovery coefficient (RC) for the 10-mm sphere of the PET/CT system used in this study is 0.55 based on our preliminary examination (Fig. 1).

Data acquisition

PET/CT scans were performed using Discovery PET/CT 600 Motion (GE Healthcare, Milwaukee, WI). Emission durations were set to $2 \mathrm{~min}$ and $20 \mathrm{~s}$. The 2-min scanned images were simulated by RG PET because our clinical scan protocol involved 10-min acquisition and was divided into 5 bins. The 20-s scanned images were simulated by DIBH PET. All images were reconstructed using a 3-dimensional ordered subset-expectation maximization (3D-OSEM) algorithm with VUE point plus and Gaussian filter. The transaxial field of view (FOV) of the reconstructed image was $550 \mathrm{~mm}$, the slice thickness was $3.27 \mathrm{~mm}$, and the matrix size was $128 \times 128$. 
The 16-slice CT scanning was performed using $120 \mathrm{kVp}, 10-200 \mathrm{~mA}$, noise index 10 , rotation time $0.6 \mathrm{~s}$, pitch 1.75:1 and slice thickness $3.75 \mathrm{~mm}$. All CT images were reconstructed by transaxial FOV $500 \mathrm{~mm}$, and the matrix size $512 \times 512$.

Data analysis and image reconstruction

For visual analysis, the PET images were evaluated by three certificated PET technologists including one nuclear medicine expert, who were engaged in PET work for more than 5 years. The images were displayed using an inverse gray scale with a SUV range of 0-4. Each sphere was scored by five grades; very good image quality 5 , sufficient good image quality 4, scarcely sufficient image quality 3, not sufficient image quality 2 , and unreadable 1 . When the visual score was $\geq 3$, it was judged as the sphere was detectable.

For physical indexes, the simulated RG PET was reconstructed using iteration-subset combinations of 2-16, 3-16, 2-32, and 5-16. Full width at half maximum (FWHM) of the Gaussian filter was changed within 3.5-7 mm for each reconstruction parameter. The smallest detectable sphere $(X \mathrm{~mm})$ was used to measure the mean SUV $\left(\mathrm{SUVm}_{\mathrm{H}, X \mathrm{~mm}}\right)$ using a region of interest (ROI) of the same diameter, and the center slice where the sphere was the most prominent was used. The BG was determined using 12 ROIs on the same slice, and the average of mean

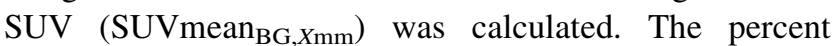
contrast $\left(Q_{\mathrm{H}, X \mathrm{~mm}}\right)$ were calculated by:

$$
\begin{aligned}
Q_{\mathrm{H}, X \mathrm{~mm}}= & \frac{\text { SUVmean }_{\mathrm{H}, X \mathrm{~mm}} / \mathrm{SUVmean}_{\mathrm{BG}, X \mathrm{~mm}}-1}{4-1} \\
& \times 100(\%) .
\end{aligned}
$$

Further, to calculate the percent BG variability $\left(N_{X \mathrm{~mm}}\right)$, 12 ROIs were set on the slice and similarly on additional four slices $( \pm 1$ and $\pm 2 \mathrm{~cm}$ of the upper and lower sides from the center slice). The $N_{X \mathrm{~mm}}$ was calculated by a total of 5 slices using average value of 60 ROIs (SUVmean $\left._{\mathrm{BG}}, \mathrm{Xmm}, 60\right)$ using the following formula:

$N_{X \mathrm{~mm}}=\frac{\mathrm{SD}_{X \mathrm{~mm}}}{\mathrm{SUVmean}_{\mathrm{BG}, X \mathrm{~mm}, 60}} \times 100(\%)$.

$\mathrm{SD}_{X \mathrm{~mm}}$ was the standard deviation (SD) of the $\mathrm{BG}$, calculated as follows:
Considering the statistical variation of PET images, $Q_{\mathrm{H}, X \mathrm{~mm}}$ and $N_{X \mathrm{~mm}}$ were calculated based on the average of three images, which were reconstructed from 0,1 and 2 min after the starting time.

With respect to the simulated DIBH PET, the images were reconstructed using iteration-subset combinations of 2-8, 2-16 and 2-32. The FWHM of the Gaussian filter was set to be $4.7 \mathrm{~mm}$. The maximum SUV (SUVmax) and radioactivity $(\mathrm{kBq} / \mathrm{mL})$ of each sphere were measured using the ROI of the same diameters from the slice where these spheres were most obviously observed. A \% difference in SUVmax (\% Dif) was defined as follows: \% Dif = simulated DIBH PET SUVmax/simulated RG PET SUVmax. The reconstruction parameters which showed similar visual and physical evaluations on the simulated RG PET were determined as the most optimal parameters for DIBH PET.

The average of each SUVmax and maximum radioactivity was calculated from the three slices which were reconstructed from the standard time and 1 and 2 min later.

\section{Clinical study}

The most optimal reconstruction parameters for DIBH and RG PET were used to examine the clinical study. Nineteen patients with a pulmonary lesion (mean $18.5 \pm 7.2 \mathrm{~mm}$, range $10-32 \mathrm{~mm}$ ) consisted of 12 males and 7 females (mean $68.8 \pm 11.9$ years, range $34-87$ years) (Table 1 ). They were examined for staging of lung cancer or for being suspect of malignancy of the lung. All patients were free from chronic obstructive pulmonary disease.

After all patients fasted at least $5 \mathrm{~h},{ }^{18} \mathrm{~F}-\mathrm{FDG}$ was injected with radioactivity of $4.4 \mathrm{MBq} / \mathrm{kg}$ (maximum dose $330 \mathrm{MBq}$ ). The RG and DIBH PET were performed at $143 \pm 11$, and $156 \pm 11 \mathrm{~min}$, respectively, after injection.

In RG PET/CT study, the respiratory motion of patients was recorded by a respiratory gating device (Varian Medical Systems, Palo Alto, CA) during the CT and PET scanning. RG CT was scanned using a cine mode, and the scan time was a breathing cycle time plus about $1 \mathrm{~s}$. This scan was repeated eight times to include an axial FOV of $154 \mathrm{~mm}$ for the PET system. The interval time between image reconstructions was set to $0.5 \mathrm{~s}$. Emission data were acquired for $10 \mathrm{~min}$ using the list-mode dynamic acquisition method. The respiratory cycles were divided into 5

$\mathrm{SD}_{X \mathrm{~mm}}=\sqrt{\sum_{k=1}^{k}\left(\mathrm{SUVmean}_{\mathrm{BG}, X \mathrm{~mm}, k}-\operatorname{SUVmean}_{\mathrm{BG}, X \mathrm{~mm}, 60}\right)^{2} /(k-1)}, \quad k=60$. 
Table 1 Characteristics of patients with pulmonary lesions

\begin{tabular}{|c|c|c|c|c|}
\hline Patient & Age & Sex & Site & $\begin{array}{l}\text { Maximum } \\
\text { diameter }(\mathrm{mm})\end{array}$ \\
\hline 1 & 72 & M & L lower lobe & 10 \\
\hline 2 & 69 & M & L lower lobe & 10 \\
\hline 3 & 76 & $\mathrm{~F}$ & $\mathrm{R}$ upper lobe & 10 \\
\hline 4 & 70 & M & L lower lobe & 10 \\
\hline 5 & 76 & M & L lower lobe & 11 \\
\hline 6 & 67 & M & L lower lobe & 12 \\
\hline 7 & 72 & $\mathrm{~F}$ & L upper lobe & 14 \\
\hline 8 & 59 & M & $\mathrm{R}$ lower lobe & 15 \\
\hline 9 & 34 & M & L upper lobe & 16 \\
\hline 10 & 60 & $\mathrm{~F}$ & $\mathrm{R}$ lower lobe & 20 \\
\hline 11 & 61 & $\mathrm{~F}$ & $\mathrm{R}$ lower lobe & 20 \\
\hline 12 & 86 & $\mathrm{~F}$ & L lower lobe & 20 \\
\hline 13 & 70 & $\mathrm{M}$ & $\mathrm{R}$ lower lobe & 22 \\
\hline 14 & 87 & $\mathrm{M}$ & $\mathrm{R}$ upper lobe & 23 \\
\hline 15 & 59 & $\mathrm{M}$ & L upper lobe & 25 \\
\hline 16 & 71 & $\mathrm{M}$ & $\mathrm{R}$ upper lobe & 25 \\
\hline 17 & 62 & $\mathrm{~F}$ & L upper lobe & 26 \\
\hline 18 & 79 & $\mathrm{~F}$ & $\mathrm{R}$ middle lobe & 30 \\
\hline 19 & 78 & $\mathrm{M}$ & L lower lobe & 32 \\
\hline
\end{tabular}

$M$ male, $F$ female, $L$ left, $R$ right

bins. The adequate bin determined to have the highest SUVmax of the lesions was chosen for RG PET SUVmax. The RG CT scanning parameters were $120 \mathrm{kVp}$, 10-100 mA, noise index 35 , rotation time $0.5 \mathrm{~s}$, and slice thickness $2.5 \mathrm{~mm}$.

In DIBH PET/CT study, after the 3-s CT scans, one bed PET acquisition for $20 \mathrm{~s}$ was repeated three times. For both the $\mathrm{CT}$ and PET acquisitions, no chest wall movement was confirmed by monitoring respiratory gating device, which indicated no misregistration between the two modalities. Among the three repeated acquisitions, the most adequate acquisition showing the highest SUVmax of the lesions was chosen for DIBH PET SUVmax. The DIBH CT scanning parameters were $120 \mathrm{kVp}, 10-200 \mathrm{~mA}$, noise index 30 , rotation time $0.6 \mathrm{~s}$, pitch $1.75: 1$, and slice thickness $3.75 \mathrm{~mm}$.

All patients provided written informed consent. Both RG and DIBH PET/CT methods were routinely performed in our institute and not intended for research. All the data were anonymized and analyzed retrospectively, and the study was approved by the security policy of the hospital.

\section{Statistical analysis}

All the data were shown using mean and SD. In the phantom study, significant differences were examined using Tukey's method. The levels of significance were set at $<0.05$. In the clinical study, correlation and Bland-Altman analyses were performed using the SUVmax of the both methods [20]. The difference in SUVmax between RG and DIBH PET was calculated, and the $95 \%$ limit of agreement was calculated by mean $\pm 1.96 \mathrm{SD}$.

\section{Result}

The most optimal reconstruction parameters for RG PET

The visual scores of hot areas regarding reconstruction parameters are shown in Table 2. Visual scores were independent from iteration-subset combinations and FWHM of the Gaussian filter if the sphere diameters were the same. When the sphere diameters were 10 and $13 \mathrm{~mm}$, the visual scores were $<3$, indicating that the spheres were not detected. Since the score of $17 \mathrm{~mm}$ of the sphere was over 4 regardless of the reconstruction parameters, the smallest detectable sphere was the $17 \mathrm{~mm}$ one.

The physical indexes were evaluated as shown in Table 3. The mean $Q_{\mathrm{H}, 17 \mathrm{~mm}}$ of $2-32$ and 5-16 was $54.8 \pm 3.2$ and $54.7 \pm 4.9 \%$, respectively. These parameters were significantly higher than those of 2-16 and 3-16 $(p<0.01)$. The mean $N_{17 \mathrm{~mm}}$ of $2-32$ and $5-16$ was $7.4 \pm 1.0$ and $8.2 \pm 0.6 \%$, respectively. The $N_{17 \mathrm{~mm}}$ of 2-32 was significantly inferior to that of 2-16 $(p<0.01)$ and 3-16 $(p<0.05)$, and the $N_{17 \mathrm{~mm}}$ of 5-16 was significantly inferior to that of 2-16 and 3-16 $(p<0.01)$. The mean $Q_{\mathrm{H}, 17 \mathrm{~mm}} / N_{17 \mathrm{~mm}}$ of 5-16 was $6.7 \pm 0.1 \%$, and it was significantly lower than those of other parameters $(p<0.01)$. Based on these results, the optimal iterationsubset combination was determined as 2-32. For the FWHM of the Gaussian filter, the $Q_{\mathrm{H}, 17 \mathrm{~mm}}$ and $N_{17 \mathrm{~mm}}$ of 2-32 did not differ significantly regardless of the FWHM of the Gaussian filter except for the $Q_{\mathrm{H}, 17 \mathrm{~mm}}$ between 3.5 and 7-mm FWHM $(p<0.05)$.

The phantom image based on the iteration-subset combination of 2-32 and 4.7-mm FWHM of the Gaussian filter is shown in Fig. 2. Both hot areas and a cold area in the center are clearly visualized, although hot areas of 10 and $13 \mathrm{~mm}$ were judged as suboptimal quality.

The most optimal reconstruction parameters for DIBH PET

The visual scores are shown in Tables 4 and 5. When the BG was filled with $1.33 \mathrm{kBq} / \mathrm{mL}$ of water, the 10 - and 13-mm spheres of the simulated DIBH PET could not be detected regardless of the reconstruction parameters. The 17-mm sphere was, however, detectable depending on the reconstruction parameters (2-8 and 2-16) although the 
Table 2 Visual scores of hot areas regarding reconstruction parameters

\begin{tabular}{|c|c|c|c|c|c|c|c|}
\hline \multirow[t]{2}{*}{ Iteration-subset } & \multirow[t]{2}{*}{ FWHM (mm) } & \multicolumn{6}{|c|}{ Sphere diameters $(\mathrm{mm})$} \\
\hline & & 10 & 13 & 17 & 22 & 28 & 37 \\
\hline \multirow[t]{4}{*}{$2-16$} & 3.5 & $2.1 \pm 1.1$ & $2.3 \pm 1.2$ & $4.4 \pm 0.5$ & 5.0 & 5.0 & 5.0 \\
\hline & 4.7 & $2.1 \pm 1.1$ & $2.4 \pm 1.2$ & $4.4 \pm 0.5$ & 5.0 & 5.0 & 5.0 \\
\hline & 5.9 & $1.9 \pm 1.2$ & $2.4 \pm 1.0$ & $4.3 \pm 0.5$ & 5.0 & 5.0 & 5.0 \\
\hline & 7 & $1.4 \pm 0.7$ & $2.6 \pm 0.7$ & $4.2 \pm 0.4$ & $4.9 \pm 0.3$ & 5.0 & 5.0 \\
\hline \multirow[t]{4}{*}{$3-16$} & 3.5 & $2.1 \pm 1.2$ & $2.8 \pm 1.1$ & $4.3 \pm 0.5$ & $4.8 \pm 0.4$ & 5.0 & 5.0 \\
\hline & 4.7 & $2.2 \pm 1.2$ & $2.7 \pm 1.0$ & $4.4 \pm 0.5$ & $4.8 \pm 0.4$ & 5.0 & 5.0 \\
\hline & 5.9 & $2.0 \pm 1.1$ & $2.8 \pm 1.2$ & $4.4 \pm 0.5$ & $4.9 \pm 0.3$ & 5.0 & 5.0 \\
\hline & 7 & $2.0 \pm 1.0$ & $2.8 \pm 0.8$ & $4.3 \pm 0.5$ & $4.8 \pm 0.4$ & 5.0 & 5.0 \\
\hline \multirow[t]{4}{*}{$2-32$} & 3.5 & $2.1 \pm 1.5$ & $2.6 \pm 1.2$ & $4.4 \pm 0.5$ & $4.8 \pm 0.4$ & 5.0 & 5.0 \\
\hline & 4.7 & $2.1 \pm 1.2$ & $2.8 \pm 1.0$ & $4.6 \pm 0.5$ & $4.8 \pm 0.4$ & 5.0 & 5.0 \\
\hline & 5.9 & $2.0 \pm 1.1$ & $2.9 \pm 1.2$ & $4.6 \pm 0.5$ & $4.9 \pm 0.3$ & 5.0 & 5.0 \\
\hline & 7 & $1.9 \pm 1.2$ & $2.7 \pm 0.9$ & $4.4 \pm 0.5$ & $4.9 \pm 0.3$ & 5.0 & 5.0 \\
\hline \multirow[t]{4}{*}{$5-16$} & 3.5 & $2.2 \pm 1.5$ & $2.8 \pm 1.4$ & $4.4 \pm 0.5$ & $4.8 \pm 0.4$ & 5.0 & 5.0 \\
\hline & 4.7 & $2.0 \pm 1.2$ & $2.8 \pm 1.2$ & $4.4 \pm 0.5$ & $4.8 \pm 0.4$ & 5.0 & 5.0 \\
\hline & 5.9 & $2.1 \pm 1.1$ & $2.8 \pm 0.8$ & $4.4 \pm 0.5$ & $4.9 \pm 0.3$ & 5.0 & 5.0 \\
\hline & 7 & $1.9 \pm 0.9$ & $2.9 \pm 0.9$ & $4.6 \pm 0.5$ & $4.9 \pm 0.3$ & 5.0 & 5.0 \\
\hline
\end{tabular}

FWHM full width at half maximum

Table 3 Physical indexes based on reconstruction parameters

\begin{tabular}{|c|c|c|c|c|c|c|}
\hline \multirow[t]{2}{*}{ Physical index } & \multirow[t]{2}{*}{ Iteration -subset } & \multicolumn{4}{|c|}{ FWHM (mm) } & \multirow[t]{2}{*}{ Mean } \\
\hline & & 3.5 & 4.7 & 5.9 & 7 & \\
\hline \multirow{4}{*}{$Q_{\mathrm{H}, 17 \mathrm{~mm}}(\%)$} & $2-16$ & $48.0 \pm 1.8$ & $45.5 \pm 3.5$ & $44.6 \pm 1.4$ & $41.1 \pm 1.4$ & $44.8 \pm 3.2$ \\
\hline & $3-16$ & $51.7 \pm 4.5$ & $49.2 \pm 4.6$ & $47.1 \pm 2.8$ & $46.0 \pm 4.7$ & $48.5 \pm 4.3$ \\
\hline & $2-32$ & $58.6 \pm 2.2$ & $54.9 \pm 2.5$ & $54.0 \pm 1.4$ & $51.7 \pm 2.1$ & $54.8 \pm 3.2^{*}$ \\
\hline & $5-16$ & $58.6 \pm 5.0$ & $56.3 \pm 5.0$ & $52.5 \pm 4.1$ & $51.1 \pm 3.7$ & $54.7 \pm 4.9^{* *}$ \\
\hline \multirow[t]{4}{*}{$N_{17 \mathrm{~mm}}(\%)$} & $2-16$ & $6.6 \pm 0.6$ & $6.3 \pm 0.4$ & $6.1 \pm 0.5$ & $5.8 \pm 0.6$ & $6.2 \pm 0.6$ \\
\hline & $3-16$ & $7.0 \pm 1.3$ & $6.5 \pm 1.3$ & $6.2 \pm 1.0$ & $5.9 \pm 1.0$ & $6.4 \pm 1.1$ \\
\hline & $2-32$ & $7.9 \pm 1.0$ & $7.5 \pm 1.3$ & $7.3 \pm 1.1$ & $7.0 \pm 1.0$ & $7.4 \pm 1.0^{\dagger}$ \\
\hline & $5-16$ & $8.7 \pm 0.8$ & $8.4 \pm 0.6$ & $7.9 \pm 0.4$ & $7.7 \pm 0.3$ & $8.2 \pm 0.6^{\dagger \dagger}$ \\
\hline \multirow[t]{4}{*}{$Q_{\mathrm{H}, 17 \mathrm{~mm}} / N_{17 \mathrm{~mm}}$} & $2-16$ & $7.2 \pm 0.8$ & $7.3 \pm 0.7$ & $7.3 \pm 0.7$ & $7.1 \pm 0.8$ & $7.2 \pm 0.1$ \\
\hline & $3-16$ & $7.4 \pm 1.5$ & $7.5 \pm 1.7$ & $7.6 \pm 1.3$ & $7.8 \pm 1.6$ & $7.6 \pm 0.2$ \\
\hline & $2-32$ & $7.5 \pm 1.0$ & $7.4 \pm 1.3$ & $7.4 \pm 1.1$ & $7.4 \pm 1.1$ & $7.4 \pm 0.0$ \\
\hline & $5-16$ & $6.8 \pm 0.8$ & $6.7 \pm 0.8$ & $6.6 \pm 0.6$ & $6.6 \pm 0.6$ & $6.7 \pm 0.1^{\S}$ \\
\hline
\end{tabular}

FWHM full width at half maximum

* The mean $Q_{\mathrm{H}, 17 \mathrm{~mm}}$ of the 2-32 was significantly higher than those of 2-16 and 3-16 $(p<0.01)$

** The mean $Q_{\mathrm{H}, 17 \mathrm{~mm}}$ of the 5-16 was significantly higher than those of 2-16 and 3-16 $(p<0.01)$

$\dagger$ The mean $N_{17 \mathrm{~mm}}$ of the 2-32 was significantly higher than those of 2-16 $(p<0.01)$ and 3-16 $(p<0.05)$

$\dagger$ The mean $N_{17 \mathrm{~mm}}$ of the 5-16 was significantly higher than those of 2-16 and 3-16 $(p<0.01)$

$\S$ The mean $Q_{\mathrm{H}, 17 \mathrm{~mm}} / N_{17 \mathrm{~mm}}$. of the 5-16 was significantly lower than those of 2-16, 3-16 and 2-32 $(p<0.01)$

scores were significantly lower than that of the simulated RG PET $(p<0.05$ and 0.01$)$. On the other hand, when the BG was filled with air, even the 10-mm sphere of the DIBH PET had sufficient image quality for all reconstruction parameters, and the visual scores were comparable to that of the RG PET.
Physical indexes were evaluated using the detectable spheres (Tables 6, 7). When the simulated DIBH PET was reconstructed by iteration-subset combinations of 2-8 and $2-16$, significant differences were not confirmed between the maximum radioactivity of the DIBH PET and that of the RG PET regardless of the BG radioactivity and sphere 


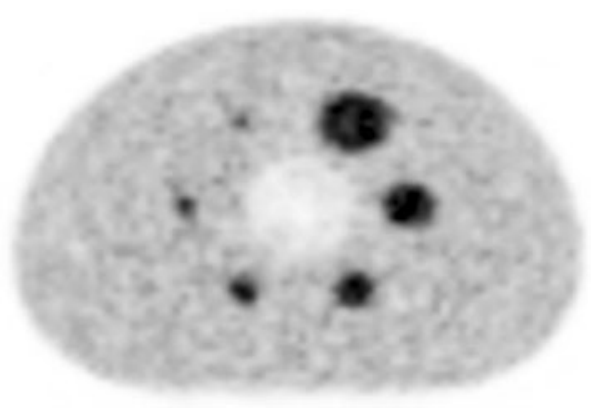

Fig. 2 The phantom image reconstructed by the iteration-subset combination of 2-32 and 4.7-mm FWHM of the Gaussian filter

diameters. However, when it was reconstructed by $2-32$, significant differences were confirmed for some spheres.

For the \% Dif, when the phantom was filled with air in the BG, the mean \% Dif of iteration-subset combinations of 2-8, 2-16 and 2-32 was $101.0 \pm 5.3,101.1 \pm 4.5$ and $103.1 \pm 5.8 \%$, respectively ( $p=$ n.s.). On the other hand, when the phantom was filled with $1.33 \mathrm{kBq} / \mathrm{mL}$ in the $\mathrm{BG}$, that of 2-8, 2-16 and 2-32 was 87.1 $\pm 11.5,107.6 \pm 5.4$ and $131.1 \pm 8.1 \%$, respectively. The $\%$ Dif of $2-16$ was significantly higher than that of 2-8 $(p<0.05)$, and was significantly lower than that of 2-32 $(p<0.05)$. Based on these results, the most optimal reconstruction parameters for DIBH PET were 2-16.

\section{Clinical study}

The mean radiation doses that patients received from DIBH and RG CT were 0.60 and $7.10 \mathrm{mSv}$, respectively. Correlation and Bland-Altman analyses are shown in Fig. 3. A regression line of the lesions was calculated as $y=-$ $0.11+1.03 x, r=0.98, p<0.000001$. For Bland-Altman analysis, the mean of RG PET SUVmax - DIBH PET SUVmax was -0.12 , and the mean \pm 1.96 SD ranged from -2.39 to 2.15 . Eighteen patients were within the mean $\pm 1.96 \mathrm{SD}$.
Table 5 Visual scores of the phantom filled with air in the BG

\begin{tabular}{llllllll}
\hline $\begin{array}{l}\text { Simulated } \\
\text { image }\end{array}$ & $\begin{array}{l}\text { Iteration- } \\
\text { subset }\end{array}$ & \multicolumn{6}{l}{ Sphere diameters $(\mathrm{mm})$} \\
\cline { 3 - 8 } & 10 & 13 & 17 & 22 & 28 & 37 \\
\hline $\mathrm{RG}$ & $2-32$ & 5 & 5 & 5 & 5 & 5 & 5 \\
DIBH & $2-8$ & $4.8 \pm 0.4$ & 5.0 & 5.0 & 5.0 & 5.0 & 5.0 \\
& $2-16$ & $4.9 \pm 0.3$ & 5.0 & 5.0 & 5.0 & 5.0 & 5.0 \\
& $2-32$ & $4.9 \pm 0.3$ & 5.0 & 5.0 & 5.0 & 5.0 & 5.0 \\
\hline
\end{tabular}

All images were reconstructed by the $4.7-\mathrm{mm}$ FWHM of the Gaussian filter

$R G$ simulated RG PET scanned for 2 min, DIBH simulated DIBH PET scanned for $20 \mathrm{~s}$

Figure 4 shows an isolated pulmonary lesion of $20 \mathrm{~mm}$ in diameter. The DIBH and RG PET SUVmax were 1.14 and 1.17 , respectively. Figure 5 shows a pulmonary lesion of $14 \mathrm{~mm}$ in diameter located close to the mediastinum. The DIBH and RG PET SUVmax were 2.66 and 3.16, respectively. These lesions could be observed not only by RG PET but also by DIBH PET. Figure 6 shows a pulmonary lesion of $11 \mathrm{~mm}$ in diameter enclosed by the circle in Fig. 3. The SUVmax of DIBH and RG PET were 10.3 and 12.6 , respectively.

\section{Discussion}

DIBH and RG PET/CT are widely performed to reduce respiratory motion artifacts. For the PET image, the image quality of DIBH PET is generally inferior to that of RG PET due to short-time acquisition. The image quality of RG PET is, however, better because of the longer time acquisition compared with that of DIBH PET. For the CT image, the image quality of DIBH CT is much superior to that of RG CT because the CT is acquired at the maximum inspiration position, which provides clear delineation like clinical CT routinely performed. The image quality of RG $\mathrm{CT}$ is, however, inferior to that of DIBH CT caused by the

Table 4 Visual scores of the phantom filled with radioactivity of $1.33 \mathrm{kBq} / \mathrm{mL}$ in the BG

\begin{tabular}{|c|c|c|c|c|c|c|c|}
\hline \multirow[t]{2}{*}{ Simulated image } & \multirow[t]{2}{*}{ Iteration-subset } & \multicolumn{6}{|c|}{ Sphere diameters (mm) } \\
\hline & & 10 & 13 & 17 & 22 & 28 & 37 \\
\hline $\mathrm{RG}$ & $2-32$ & $4.0 \pm 1.1$ & $4.2 \pm 1.2$ & $4.7 \pm 0.7$ & 5 & 5 & 5 \\
\hline \multirow[t]{3}{*}{ DIBH } & $2-8$ & $1.4 \pm 0.7$ & $2.2 \pm 0.7$ & $3.7 \pm 0.5^{* *}$ & $4.7 \pm 0.5$ & $4.9 \pm 0.3$ & 5.0 \\
\hline & $2-16$ & $1.7 \pm 0.9$ & $2.4 \pm 0.5$ & $3.6 \pm 0.5^{*}$ & $4.2 \pm 0.7$ & $4.7 \pm 0.5$ & 5.0 \\
\hline & $2-32$ & $1.3 \pm 0.5$ & $1.9 \pm 0.6$ & $2.8 \pm 0.8^{*}$ & $3.9 \pm 0.9 * *$ & $4.3 \pm 0.7$ & $4.9 \pm 0.3$ \\
\hline
\end{tabular}

All images were reconstructed by the 4.7-mm FWHM of the Gaussian filter. The 10 and 13-mm spheres cf simulated DIBH PET could not be detected regardless of the reconstruction parameters

$R G$ simulated RG PET scanned for 2 min, DIBH simulated DIBH PET scanned for $20 \mathrm{~s}$

The score of simulated DIBH PET was significantly lower than that of simulated RG PET $(* p<0.01, * * p<0.05)$ 
Table 6 Physical indexes for the phantom filled with radioactivity of $1.33 \mathrm{kBq} / \mathrm{mL}$ in the BG

\begin{tabular}{|c|c|c|c|c|c|c|c|c|c|}
\hline \multirow{2}{*}{$\begin{array}{l}\text { Simulated } \\
\text { image }\end{array}$} & \multirow{2}{*}{$\begin{array}{l}\text { Iteration- } \\
\text { subset }\end{array}$} & \multirow[t]{2}{*}{ Parameter } & \multicolumn{6}{|c|}{ Sphere diameters (mm) } & \multirow[t]{2}{*}{ Mean } \\
\hline & & & 10 & 13 & 17 & 22 & 28 & 37 & \\
\hline RG & $2-32$ & $\operatorname{Max}(\mathrm{kBq} / \mathrm{mL})$ & & & $9.7 \pm 1.3$ & $12.0 \pm 0.6$ & $11.9 \pm 0.4$ & $12.8 \pm 0.3$ & \\
\hline \multirow[t]{6}{*}{ DIBH } & $2-8$ & $\operatorname{Max}(\mathrm{kBq} / \mathrm{mL})$ & & & $7.3 \pm 0.6$ & $10.1 \pm 0.5$ & $10.3 \pm 0.5$ & $13.2 \pm 0.7$ & \\
\hline & & $\%$ Dif & & & $74.4 \pm 11.8$ & $85.4 \pm 5.8$ & $86.2 \pm 5.6$ & $102.4 \pm 6.3$ & $87.1 \pm 11.5$ \\
\hline & $2-16$ & $\operatorname{Max}(\mathrm{kBq} / \mathrm{mL})$ & - & - & $10.3 \pm 0.9$ & $12.5 \pm 1.4$ & $12.4 \pm 0.8$ & $14.8 \pm 0.4$ & \\
\hline & & $\%$ Dif & & & $106.4 \pm 17.0$ & $104.9 \pm 13.1$ & $103.7 \pm 7.4$ & $115.6 \pm 4.4$ & $107.6 \pm 5.4^{\dagger}$ \\
\hline & $2-32$ & $\operatorname{Max}(\mathrm{kBq} / \mathrm{mL})$ & & & $13.8 \pm 1.8^{* *}$ & $14.9 \pm 2.8$ & $15.0 \pm 1.6^{* *}$ & $16.8 \pm 1.3 *$ & \\
\hline & & $\%$ Dif & & & $142.5 \pm 26.4$ & $125.4 \pm 24.5$ & $125.3 \pm 14.4$ & $131.1 \pm 10.7$ & $131.1 \pm 8.1^{\dagger \dagger}$ \\
\hline
\end{tabular}

All images were reconstructed by the 4.7-mm FWHM of the Gaussian filter

$R G$ simulated RG PET scanned for 2 min, DIBH simulated DIBH PET scanned for $20 \mathrm{~s}$, Max maximum radioactivity (kBq/mL), \% Dif simulated DIBH PET SUVmax/simulated RG PET SUVmax $\times 100(\%)$

The Max of simulated DIBH PET was significantly higher than that of simulated RG PET $(* p<0.01, * * p<0.05)$

$\dagger$ The mean \% Dif of 2-16 was significantly higher than that of 2-8 $(p<0.05)$

+ The mean $\%$ Dif of 2-32 was significantly higher than that of 2-16 $(p<0.05)$

Table 7 Physical indexes for the phantom filled with air in the BG

\begin{tabular}{|c|c|c|c|c|c|c|c|c|c|}
\hline \multirow{2}{*}{$\begin{array}{l}\text { Simulated } \\
\text { image }\end{array}$} & \multirow{2}{*}{$\begin{array}{l}\text { Iteration- } \\
\text { subset }\end{array}$} & \multirow[t]{2}{*}{ Parameter } & \multicolumn{6}{|c|}{ Sphere diameters $(\mathrm{mm})$} & \multirow[t]{2}{*}{ Mean } \\
\hline & & & 10 & 13 & 17 & 22 & 28 & 37 & \\
\hline RG & $2-32$ & $\begin{array}{l}\operatorname{Max}(\mathrm{kBq} / \\
\mathrm{mL})\end{array}$ & $7.0 \pm 0.8$ & $9.6 \pm 0.4$ & $9.9 \pm 0.4$ & $10.4 \pm 0.1$ & $11.1 \pm 0.3$ & $11.5 \pm 0.4$ & \\
\hline \multirow[t]{3}{*}{ DIBH } & $2-8$ & $\begin{array}{l}\text { Max (kBq/ } \\
\text { mL) } \\
\text { \% Dif }\end{array}$ & $\begin{array}{r}6.4 \pm 0.3 \\
90.8 \pm 9.7\end{array}$ & $\begin{array}{r}9.8 \pm 0.3 \\
101.9 \pm 5.5\end{array}$ & $\begin{array}{r}10.4 \pm 0.1 \\
105.8 \pm 3.9\end{array}$ & $\begin{array}{r}10.9 \pm 0.1 \\
104.5 \pm 2.0\end{array}$ & $\begin{array}{r}11.3 \pm 0.3 \\
102.3 \pm 3.2\end{array}$ & $\begin{array}{r}11.7 \pm 0.4 \\
100.9 \pm 5.2\end{array}$ & $101.0 \pm 5.3$ \\
\hline & $2-16$ & $\begin{array}{l}\text { Max (kBq/ } \\
\text { mL) } \\
\text { \% Dif }\end{array}$ & $\begin{array}{c}6.6 \pm 0.6 \\
93.8 \pm 12.8\end{array}$ & $\begin{array}{c}9.4 \pm 0.9 \\
97.8 \pm 10.3\end{array}$ & $\begin{array}{r}10.0 \pm 0.2 \\
101.8 \pm 4.1\end{array}$ & $\begin{array}{r}10.9 \pm 0.3 \\
104.8 \pm 3.2\end{array}$ & $\begin{array}{r}11.6 \pm 0.5 \\
105.2 \pm 4.9\end{array}$ & $\begin{array}{r}11.9 \pm 0.3 \\
103.1 \pm 4.4\end{array}$ & $101.1 \pm 4.5$ \\
\hline & $2-32$ & $\begin{array}{l}\text { Max }(\mathrm{kBq} / \\
\mathrm{mL}) \\
\text { \% Dif }\end{array}$ & $\begin{array}{c}6.8 \pm 0.5 \\
97.4 \pm 12.1\end{array}$ & $\begin{array}{c}9.7 \pm 1.0 \\
100.4 \pm 11.3\end{array}$ & $\begin{array}{r}9.5 \pm 0.2 \\
96.0 \pm 3.8\end{array}$ & $\begin{array}{r}11.3 \pm 0.6 \\
108.3 \pm 6.0\end{array}$ & $\begin{array}{r}11.8 \pm 0.3 \\
107.2 \pm 3.0\end{array}$ & $\begin{aligned} 12.5 & \pm 0.2^{* *} \\
109.1 & \pm 4.1\end{aligned}$ & $103.1 \pm 5.8$ \\
\hline
\end{tabular}

All images were reconstructed by the 4.7-mm FWHM of the Gaussian filter

$R G$ simulated RG PET scanned for 2 min, DIBH simulated DIBH PET scanned for $20 \mathrm{~s}$, Max maximum radioactivity (kBq/mL), \% Dif simulated DIBH PET SUVmax/simulated RG PET SUVmax $\times 100(\%)$

The Max of simulated DIBH PET was significantly higher than that of simulated RG PET $(* * p<0.05)$

Fig. 3 Correlation relationship (left) and Bland-Altman analysis (right) between RG and DIBH PET SUVmax. High correlation and little dispersion were observed. *This dot consisted of two lesions having very similar SUVmax. A case enclosed by the circle in the Bland-Altman analysis is shown in Fig. 6
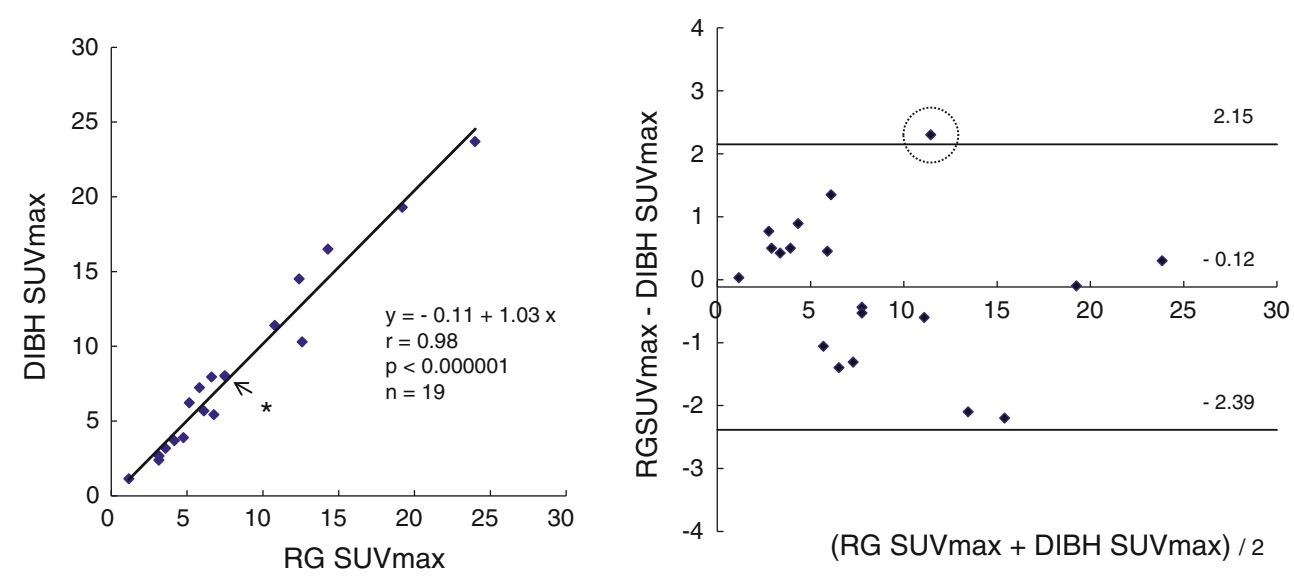


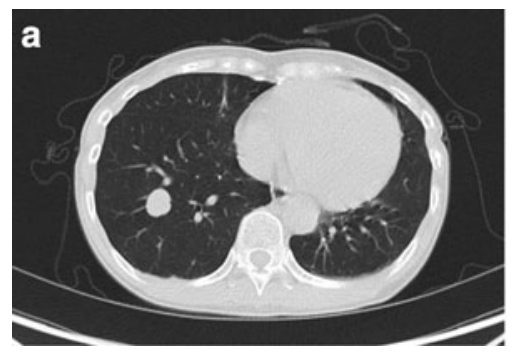

b

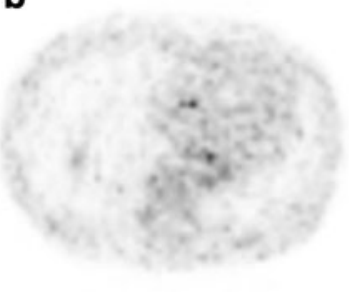

C

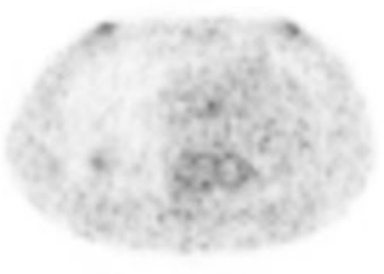

Fig. 4 A 60-year-old female patient with an isolated pulmonary lesion in the right lower lobe. DIBH CT (a), DIBH PET (b), and RG PET (c)

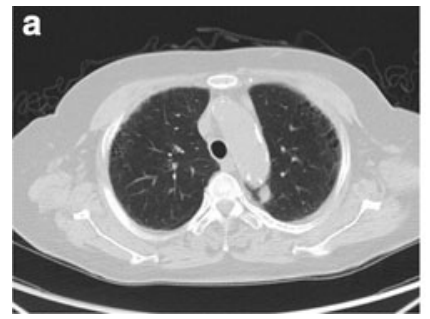

b

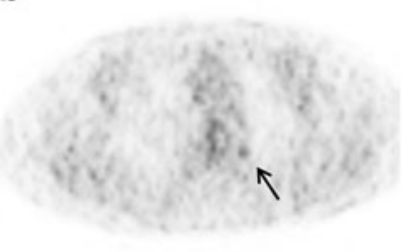

C

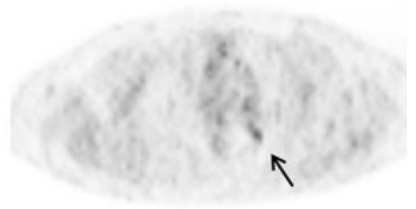

Fig. 5 A 72-year-old female patient with a pulmonary lesion located close to the mediastinum in the left upper lobe (arrow). DIBH CT (a), DIBH PET (b), and RG PET (c)

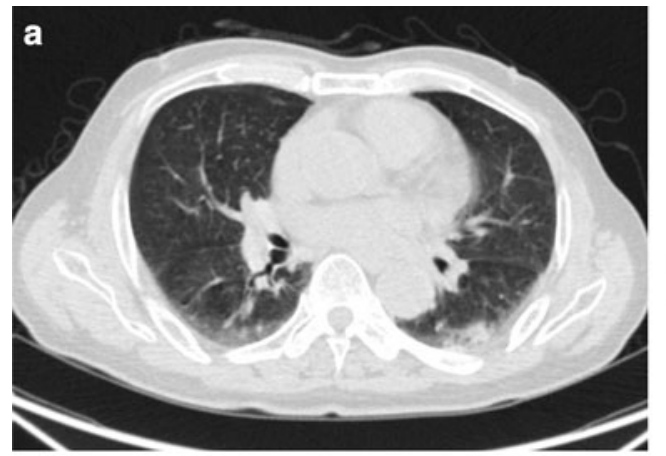

b

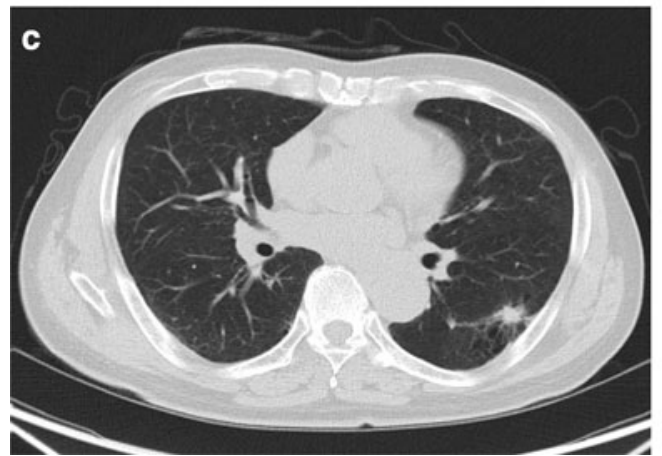

d
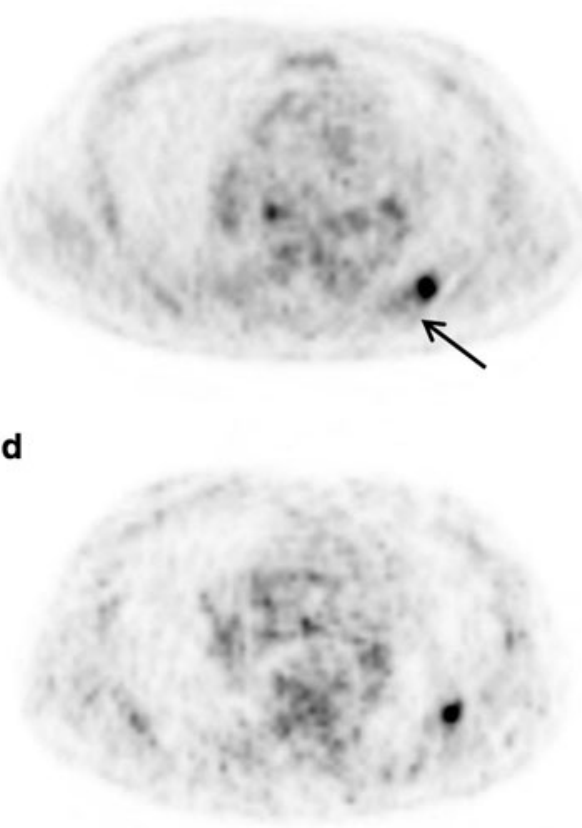

Fig. 6 A 76-year-old male patient with a pulmonary lesion in the left lower lobe. RG CT (a), RG PET (b), DIBH CT (c), and DIBH PET (d). The RG PET SUVmax was increased by the poorly inflated dorsal lung with relatively high radioactivity (arrow)

body motion of free breathing and low tube current time product. DIBH PET/CT has significant advantages in terms of rapid examination and low radiation exposure. While about $15 \mathrm{~min}$ of RG PET/CT examination was needed, $<5$ min was necessary for DIBH PET/CT. In terms of radiation doses, RG CT was about 12 times higher than that of DIBH CT. Therefore, we compared the detectability between DIBH and RG PET, and the limitation and indication for DIBH PET were evaluated.

For DIBH PET acquisition time, Miyashita et al. [17] reported that optimum emission time of the DIBH PET technique greater than $90 \mathrm{~s}$ acquisition is preferable for 
clinical use. However, the optimal acquisition time varied according to PET systems, and a long acquisition time and repeated acquisition were not acceptable for patients with pulmonary lesions as Kawano et al. [13] noted in a clinical setting that using DIBH PET with a breath holding of $<30$ s could be helpful. The PET system used in this study has high sensitivity (3D: $9.1 \mathrm{cps} / \mathrm{kBq}$ ). Torizuka et al. [12] have reported that a single $20 \mathrm{~s}$ acquisition of breath-hold PET/CT enabled more precise measurement of tumor ${ }^{18} \mathrm{~F}$ FDG uptake. DIBH PET acquisition time was, therefore, set to be $20 \mathrm{~s}$, which might be the clinical upper limit of breath holding.

The most optimal reconstruction parameters for DIBH and RG PET were determined in the phantom study, and those of RG PET were regarded as a gold standard. The $Q_{\mathrm{H}, 17 \mathrm{~mm}}$ was more important than the $N_{17 \mathrm{~mm}}$ because most of the pulmonary lesions were surrounded by low BG radioactivity. The optimal iteration-subset combination was found to be 2-32 based on high $Q_{\mathrm{H}, 17 \mathrm{~mm}}$ and $Q_{\mathrm{H}, 17 \mathrm{~mm}} /$ $\mathrm{N}_{17 \mathrm{~mm}}$. To determine the FWHM of the Gaussian filter, since the $Q_{\mathrm{H}, 17 \mathrm{~mm}}$ and $N_{17 \mathrm{~mm}}$ of 2-32 did not differ significantly among filter types, we selected 4.7-mm FWHM in accordance with our clinical parameter. Pulmonary lesions located close to the mediastinum were simulated using the phantom filled with $1.33 \mathrm{kBq} / \mathrm{mL}$ in the $\mathrm{BG}$, which is similar to clinical conditions. The lesions surrounded by pulmonary region were simulated using the phantom filled with air in the BG. Even the 10-mm sphere was clearly visualized when those phantom images were reconstructed by this parameters. Consequently, it was judged that the reconstruction parameters, acquisition time and number of bins for RG PET were appropriate.

The optimal reconstruction parameters of DIBH PET were different from that of RG PET, and reconstruction parameters of DIBH PET could be obtained by reducing the number of subsets for those of RG PET in the state of fixing the number of iterations. The reason for this was that the images with low count such as DIBH PET may have been diverged when the number of subsets was high [21]. Using the same reconstruction parameters as that of RG PET, the maximum radioactivity of the simulated DIBH PET was significantly higher for some spheres compared to that of the simulated RG PET.

The clinical study was performed using each of the most optimal reconstruction parameters. In 19 cases, high correlation and little dispersion were observed between DIBH and RG PET SUVmax. In our study, since most of the lesions were surrounded by low pulmonary radioactivity, the overlapped radioactivity from the mediastinum was negligible. This result was consistent with that of the phantom study filled with air in the BG. Even the 10-mm sphere showed equivalent image quality between the simulated DIBH PET and RG PET.
The DIBH PET showed high contrast between the lesion and the BG because the lung was filled with a significant amount of air for maximum inspiration during breath holding. The lesion was visible as low as SUVmax of 1.1, and DIBH and RG PET SUVmax had nearly equivalent values. When the lesion was located close to the mediastinum, DIBH and RG PET also had nearly equivalent image quality since the major part of the lesion was surrounded by low pulmonary radioactivity. In Fig. 6, the high SUVmax was obtained even in a small lesion. Several articles have reported that the SUVmax of the lesions which are small and located in the lower lung is especially decreased by the respiratory motion artifact under free breathing [11-13]. Since the lesion in this patient was also small and in the lower lobe, the underestimation of SUVmax was highly improved by DIBH and RG PET/CT. However, the difference between DIBH and RG PET SUVmax was the highest in the clinical study. The RG PET SUVmax was overestimated because the lesion was surrounded by poorly inflated dorsal lung with relatively high radioactivity (SUVmean 1.76). Using the DIBH PET/CT, the dorsal vascular shadow was not found. This case has shown that DIBH PET could provide higher accurate SUVmax than that of RG PET. Regarding CT image quality, the DIBH CT could clearly describe the lesion, but the RG CT could not.

This study has limitations. As shown in the phantom study filled with $1.33 \mathrm{kBq} / \mathrm{mL}$ in the $\mathrm{BG}$, the sphere size $<17 \mathrm{~mm}$ could not be detected. The equivalent image quality between DIBH and RG PET might not be obtained according to the BG radioactivity around the lesion. The detectability of lesions located close to the mediastinum could be limited depending on their sizes and accumulations. All of the lesions surrounded by the pulmonary region may not provide equivalent image quality between DIBH and RG PET as shown in Fig. 6. Further clinical assessment is indicated in this respect.

DIBH PET/CT has a significant practical value, but poor signal-to-noise ratio caused by the size, uptake and target to BG contrast of the lesions. RG PET/CT and DIBH PET/ CT under multiple summed acquisition methods reported by Nehmeh et al. [14-17] are preferable to assess the lesions which are difficult for detecting using DIBH PET.

\section{Conclusion}

DIBH PET/CT may be the most practical method which can be the first choice to reduce respiratory motion artifacts if the detectability of DIBH PET is equivalent with that of RG PET. Although DIBH PET may have limitations in suboptimal signal-to-noise ratio due to the short-time acquisition, most of the lesions surrounded by low BG 
radioactivity could provide nearly equivalent image quality between DIBH and RG PET studies when each of the most optimal reconstruction parameters was used.

Conflict of interest The authors declare no conflict of interest.

Open Access This article is distributed under the terms of the Creative Commons Attribution License which permits any use, distribution, and reproduction in any medium, provided the original author(s) and the source are credited.

\section{References}

1. Bar-Shalom R, Yefremov N, Guralnik L, Gaitini D, Frenkel A, Kuten A, et al. Clinical performance of PET/CT in evaluation of cancer: additional value for diagnostic imaging and patient management. J Nucl Med. 2003;44(8):1200-9.

2. Tsukamoto E, Ochi S. PET/CT today: system and its impact on cancer diagnosis. Ann Nucl Med. 2006;20(4):255-67.

3. Gerth HU, Juergens KU, Dirksen U, Gerss J, Schober O, Franzius C. Significant benefit of multimodal imaging: PET/CT compared with PET alone in staging and follow-up of patients with Ewing tumors. J Nucl Med. 2007;48(12):1932-9.

4. Nakamoto Y, Osman M, Cohade C, Marshall LT, Links JM, Kohlmyer S, et al. PET/CT: comparison of quantitative tracer uptake between germanium and CT transmission attenuationcorrected images. J Nucl Med. 2002;43(9):1137-43.

5. Bettinardi V, Picchio M, Di Muzio N, Gianolli L, Messa C, Gilardi MC. PET/CT for radiotherapy: image acquisition and data processing. Q J Nucl Med Mol Imaging. 2010;54(5):455-75.

6. Pommier P, Touboul E, Chabaud S, Dussart S, Le Pechoux C, Giammarile F, et al. Impact of (18)F-FDG PET on treatment strategy and 3D radiotherapy planning in non-small cell lung cancer: a prospective multicenter study. AJR Am J Roentgenol. 2010;195(2):350-5.

7. Cerfolio RJ, Bryant AS, Winokur TS, Ohja B, Bartolucci AA. Repeat FDG-PET after neoadjuvant therapy is a predictor of pathologic response in patients with non-small cell lung cancer. Ann Thorac Surg. 2004;78(6):1903-9.

8. Yamamoto Y, Kameyama R, Murota M, Bandoh S, Ishii T, Nishiyama Y. Early assessment of therapeutic response using FDG PET in small cell lung cancer. Mol imaging Biol. 2009;11(6): 467-72.
9. Nehmeh SA, Erdi YE, Ling CC, Ling CC, Rosenzweig KE, Squire OD, et al. Effect of respiratory gating on reducing lung motion artifacts in PET imaging of lung cancer. Med Phys. 2002;29(3):366-71.

10. Nehmeh SA, Erdi YE, Pan T, Pevsner A, Rosenzweig KE, Yorke E, et al. Four-dimensional (4D) PET/CT imaging of the thorax. Med Phys. 2004;31(12):3179-86.

11. Kawano T, Ohtake E, Inoue T. Deep-inspiration breath-hold PET/CT of lung cancer: maximum standardized uptake value analysis of 108 patients. J Nucl Med. 2008;49(8):1223-31.

12. Torizuka T, Tanizaki Y, Kanno T, Futatsubashi M, Yoshikawa E, Okada H, et al. Single 20-second acquisition of deep-inspiration breath-hold PET/CT: clinical feasibility for lung cancer. J Nucl Med. 2009;50(10):1579-84.

13. Kawano T, Ohtake E, Inoue T. Deep-inspiration breath-hold $\mathrm{PET} / \mathrm{CT}$ versus free breathing PET/CT and respiratory gating PET for reference: evaluation in 95 patients with lung cancer. Ann Nucl Med. 2011;25(2):109-16.

14. Nehmeh SA, Erdi YE, Meirelles GS, Squire O, Larson SM, Humm JL, et al. Deep-inspiration breath-hold PET/CT of the thorax. J Nucl Med. 2007;48(1):22-6.

15. Meirelles GS, Erdi YE, Nehmeh SA, Squire OD, Larson SM, Humm JL, et al. Deep-inspiration breath-hold PET/CT: clinical findings with a new technique for detection and characterization of thoracic lesions. J Nucl Med. 2007;48(5):712-9.

16. Daisaki H, Shinohara H, Terauchi T, Murano T, Shimada N, Moriyama N, et al. Multi-bed-position acquisition technique for deep inspiration breath-hold PET/CT: a preliminary result for pulmonary lesions. Ann Nucl Med. 2010;24(3):179-88.

17. Miyashita K, Tateishi U, Nishiyama Y, Minamimoto R, Shizukuishi K, Inoue T. Optimum emission time in deep-inspiration breath-hold PET-CT: a preliminary result. Ann Nucl Med. 2010;24(7):559-63.

18. Fukukita H, Senda M, Terauchi T, Suzuki K, Daisaki H, Matsumoto K, et al. Japanese guideline for the oncology FDG-PET/ CT data acquisition protocol: synopsis of Version 1.0. Ann Nucl Med. 2010;24(4):325-34.

19. Mejia AA, Nakamura T, Masatoshi I, Hatazawa J, Masaki M, Watanuki S. Estimation of absorbed doses in humans due to intravenous administration of fluorine-18-fluorodeoxyglucose in PET studies. J Nucl Med. 1991;32(4):699-706.

20. Bland M, Altman DG. Statistical methods for assessing agreement between two methods of clinical measurement. Lancet. 1986;1(8476):307-10.

21. Maru S, Yanagisawa M. Basic evaluation of OSEM algorithm by assessing iteration times and number of subsets in a hot spot phantom study. J Radiol Technol. 2001;57(10):1233-9. 